\title{
Research Note \\ The proper motion of the Magellanic Clouds: The UCAC2-Hipparcos inconsistency
}

\author{
Y. Momany ${ }^{1}$ and S. Zaggia ${ }^{2}$ \\ 1 Dipartimento di Astronomia, Università di Padova, Vicolo dell’Osservatorio 2, 35122 Padova, Italy \\ e-mail: momany@pd.astro.it \\ 2 INAF - Osservatrio Astronomico di Trieste, via Tiepolo 11, 34131 Trieste, Italy
}

Received 2 November 2004 / Accepted 28 March 2005

\begin{abstract}
Using the USNO CCD Astrograph all-sky Catalog (UCAC2), we measure the mean proper motion of the two Magellanic Clouds. Appropriately-selected LMC populations show a proper motion $\langle\mu \alpha, \mu \delta\rangle \simeq(+0.84,+4.32)$ that is significantly higher, in $\langle\mu \delta\rangle$, than currently accepted Hipparcos-like values; $\langle\mu \alpha, \mu \delta\rangle \simeq(+1.94,-0.14)$. A higher $\langle\mu \alpha\rangle$ value is also found for the SMC. Interestingly, the mean UCAC2 LMC proper motion agrees very well with the only work in the literature (Anguita et al. 2000, AJ, 120, 845) pointing to an unbound Magellanic Clouds-Milky Way interaction. Nonetheless, the implications of the UCAC2 proper motion are hard to reconcile with our present day understanding of the Clouds-Galaxy interaction unless one assumes a more massive Milky Way. Consequently, although no sources of systematic error have been identified, it is perhaps most likely that the UCAC2 catalog has an as yet unidentified systematic error resulting in an inconsistency between UCAC2 and Hipparcos based results for the Magellanic Clouds.
\end{abstract}

Key words. astronomical data bases: miscellaneous - astrometry - galaxies: Magellanic Clouds - galaxies: interactions

\section{Introduction}

The gas envelope surrounding both the Large and Small Magellanic Clouds (LMC and SMC) is reminiscent of past interactions between the two galaxies. In turn, the Magellanic Stream is evidence of past interactions of both galaxies with the Milky Way (Kroupa \& Bastian 1997). Modeling the past stellar and dynamical evolution of the Magellanic Clouds requires good knowledge of many parameters, in particular those concerning their spatial velocity vector and transverse velocity ( $v_{\mathrm{t}}$, van der Marel 2004). A sound determination of the latter parameter holds the key for a better understanding of the Milky Way-Magellanic Clouds interaction. The LMC/SMC transverse velocity can be measured directly from the observed proper motion of the galaxies, or alternatively, via (i) dynamical modeling of the Magellanic Stream; or (ii) analysis of the line-of-sight velocity field of the LMC/SMC.

Many groups have addressed the $v_{t}$ measure of the Magellanic Clouds via the direct method of proper-motion (pm). Being closer, the LMC has been the subject of more detailed studies (see Table 1 for a compilation of pm values). At first sight, one notes an overall excellent agreement between the first 6 determinations. However, there is one outlier ( $\sim 5.5 \sigma$ times the average value in declination), namely that of Anguita, Loyola and Pedreros (2000, hereafter ALP00). The ALP00 study is based on 125 CCD images spanning 8 years, and put on a QSO absolute reference frame. It was the first pm analysis of $\mathrm{LMC}$ stars reaching $V \simeq 22$; i.e. including nearly all its composite stellar populations.

In a later work, Pedreros et al. (2002) using a similar CCD data set and the same QSO method, contradicted their earlier ALP00 results and derived Hipparcos-consistent pm values. Puzzled by this discrepancy, Pedreros et al. (2002) searched for systematic errors possibly affecting the ALP00 study. Their most plausible explanation pointed to peculiar pm or peculiarities in the rotation curve of the LMC between their two studied fields (located at different positions and distances from the LMC center). Further progress on this issue awaited results from the 9 million LMC stars of the MACHO project. To our knowledge, the only MACHO study on the LMC pm is that of Drake et al. (2002), confirming "normal" pm values. Thus, the ALP00 study remains the only, inconsistent, pm value pointing to the Magellanic Clouds being unbound.

Following the methodology outlined in Momany et al. (2004) we analyze UCAC2 (Zacharias et al. 2004) pm data of the Magellanic Clouds. Interestingly, we find that the UCAC2 data unequivocally point toward a high $\langle\mu \delta\rangle$ of the LMC, as in the ALP00 study. In this paper we attempt to assess the reliability of the UCAC2 pm data by searching for color/magnitude dependencies or other systematic effects. The UCAC2 pm data show no significant evidence of systematic errors. 
Table 1. A compilation of LMC proper motion studies.

\begin{tabular}{llll}
\hline \hline Source & $\begin{array}{l}\mu \alpha \\
\left(\mathrm{mas} \mathrm{yr}^{-1}\right)\end{array}$ & $\begin{array}{l}\mu \delta \\
\left(\mathrm{mas} \mathrm{yr}^{-1}\right)\end{array}$ & System \\
\hline Kroupa et al. (1994) & $+1.30 \pm 0.60$ & $+1.10 \pm 0.70$ & PPM \\
Jones et al. (1994) & $+1.20 \pm 0.28$ & $+0.26 \pm 0.27$ & Galaxies \\
Jones et al. (1994) & $+1.37 \pm 0.28$ & $-0.18 \pm 0.27$ & Galaxies \\
Kroupa Bastian (1997) & $+1.94 \pm 0.29$ & $-0.14 \pm 0.36$ & Hipparcos \\
Drake et al. (2002) $)^{\dagger}$ & $+1.40 \pm 0.40$ & $+0.38 \pm 0.25$ & MACHO \\
Pedreros et al. (2002) $)^{\dagger}$ & $+2.00 \pm 0.20$ & $+0.40 \pm 0.20$ & QSO \\
\hline Anguita et al. (2000) & $+1.70 \pm 0.20$ & $+2.90 \pm 0.20$ & QSO \\
\hline
\end{tabular}

$\dagger$ The reported pm are those corrected and listed in van der Marel (2002).

The Hipparcos-UCAC2 discrepancy basically remains unsolved. The plausibility and implications of the UCAC2 and ALP00 values is discussed.

\section{The UCAC2 LMC and SMC proper motion}

The recent availability of large, high precision astrometric and photometric catalogs like 2MASS (Cutri et al. 2003), SPM3 (Girard et al. 2004) and UCAC2 (Zacharias et al. 2004) allow kinematic investigations of structures within the Galaxy and determination of the orbits of its nearby satellite companions. In Momany et al. (2004), using the UCAC2 data, we have been able to recover/confirm the HST pm measurement of the Sagittarius dwarf galaxy located at a distance of $25 \mathrm{kpc}$ : $\langle\mu \alpha, \mu \delta\rangle \simeq(-2.5,-2.0) \mathrm{mas} / \mathrm{yr}$ (Irwin et al. 1996). This value has also been recently confirmed by Dinescu et al. (2004) using the SPM3 catalog. Thus, the Sagittarius dwarf is an excellent example of the reliability of UCAC2 pm data, and encourages the use of these data for a similar analysis for the Magellanic Clouds.

Combining the 2MASS infrared photometry with UCAC2 optical and pm data offers a unique opportunity to discriminate LMC/SMC stellar populations from the Galactic (disk/halo) contribution. On the one hand, this will help identify possible dependencies of the pm data on the employed photometric magnitudes and colors, and on the other, it dramatically expands the number of pm stars in different LMC/SMC populations (having different colors and magnitudes). This reduces the error budget and possible contamination effects. The net result is a more robust estimate of the bulk pm. Zacharias et al. estimate UCAC2 pm errors to be 1-3 mas/yr for 12th mag stars and about 4-7 mas/yr at 16 th mag. Throughout this paper, we use absolute pm data for LMC and SMC stellar populations having $12 \leq R \leq 16$. Following a $3 \sigma$ clipping, we estimate the mean $\mathrm{rms}$ of the average pm values to be $\leq 4.0 \mathrm{mas} / \mathrm{yr}$. Although quite significant, such an rms error is acceptable considering the distance of the Magellanic Clouds. Moreover, we prefer to assume such a high error rather than that obtained by dividing it by the square root of the number of stars.

The first epoch data for the pm come from different catalogs including Hipparcos/Tycho, AC2000.2, as well as re-measured AGK2, NPM and SPM plates. As in Momany et al. (2004), we limit the UCAC2 pm data to only one first epoch catalog in order to limit internal inhomogeneities, namely the AC2000.2 catalog (Urban et al. 1998, 2001). Based on the Carte $\mathrm{du}$ Ciel Astrographic plates and measurements, the construction of the AC2000.2 was performed scanning all the collected plates and then collecting all the positional measurements in a homogeneous system based on Hipparcos. A key feature of AC2000.2 is the mean epoch of the positions which range from 1890 to 1940 . In the particular case of the LMC the mean epoch of AC2000.2 is $\simeq 1893.5$. Comparing the LMC mean epoch of AC2000.2 with the observational epoch of the UCAC2 (1998.1 $\div 2002.9)$ gives a very large epoch span for the pm measurements, therefore reducing the pm errors.

Figure 1 summarizes our analysis in deriving the LMC (upper panels) and SMC (lower panels) mean pm. The left panels of Fig. 1 show combined UCAC2/2MASS $R,(R-K)$ colormagnitude diagrams, for two fields $\left(1^{\circ}\right.$ radius each) centered on the LMC and SMC. Dark symbols are stars whose pm originates only from the AC2000.2 catalog (and used in estimating the $\mathrm{pm}$ ), whereas light symbols have pm from a number of different catalogs, including Hipparcos. The middle panels highlight the selected LMC/SMC stellar populations. Relying on the color-magnitude diagrams in Nikoleav \& Weinberg (2000) and Alcock et al. (2000) of the LMC, one can easily disentangle LMC stellar populations from the foreground Galactic contribution. Indeed, the two left-most selected populations (open triangles) are almost certainly LMC young main sequence and Helium burning stars. On the redder side, the three right-most populations (open circles) are red super-giants, oxygen- and carbon-rich LMC stars, respectively. In between the LMC populations, one identifies two vertical sequences (open squares) which are Galactic disk F-K dwarfs and K giants. As noted by Alcock et al. (2000), in these regions of the color-magnitude diagram the Galactic disk stars out-number those belonging to both the Thick Disk and Halo combined. A confirmation of this comes from a Besançon synthetic color-magnitude diagram of the Galactic contribution: modeling in both the LMC and SMC directions clearly showed that virtually no Galactic stars fall in the Magellanic Clouds selected regions. Filled circles are 35 Hipparcos stars tabled in Kroupa \& Bastian (1997, their Table 5 excluding star number 26222). Hipparcos stars plotted as open circles are those falling inside our $1^{\circ}$ radius UCAC2 fields.

The right panels show the averaged $3 \sigma$ clipped $\langle\mu \alpha, \mu \delta\rangle$ diagram of all selected populations. For the LMC (upper panel) we easily distinguish three separate groups, namely: (i) the Galactic populations characterized by a high $\langle\mu \delta\rangle \simeq$ $8.5 \mathrm{mas} / \mathrm{yr}$; (ii) the LMC populations clearly clustering around $\langle\mu \alpha, \mu \delta\rangle \simeq(0.9,3.9) \mathrm{mas} / \mathrm{yr}$; and (iii) the Hipparcos measurements at $\langle\mu \alpha, \mu \delta\rangle \simeq(1.5,-0.3)$ mas/yr. Likewise for the SMC (lower panel): (i) the Galactic populations at $\langle\mu \alpha, \mu \delta\rangle \simeq$ $(9.0,-1.5) \mathrm{mas} / \mathrm{yr}$; (ii) the SMC populations at $\langle\mu \alpha, \mu \delta\rangle \simeq$ $(4.5,-3.3) \mathrm{mas} / \mathrm{yr}$; and (iii) the Hipparcos measurements at $\langle\mu \alpha, \mu \delta\rangle \simeq(1.0,-1.0) \mathrm{mas} / \mathrm{yr}$. The right panels of Fig. 1 show the discrepancy between the Hipparcos and UCAC2 pm mean values, mainly in $\langle\mu \delta\rangle$ for the LMC and in both directions for the SMC. Overall, the UCAC2 data indicate a mean pm for the $\mathrm{LMC}$ of $\langle\mu \alpha, \mu \delta\rangle \simeq(0.84,+4.32) \mathrm{mas} / \mathrm{yr}$ based on $\sim 1620$ stars 


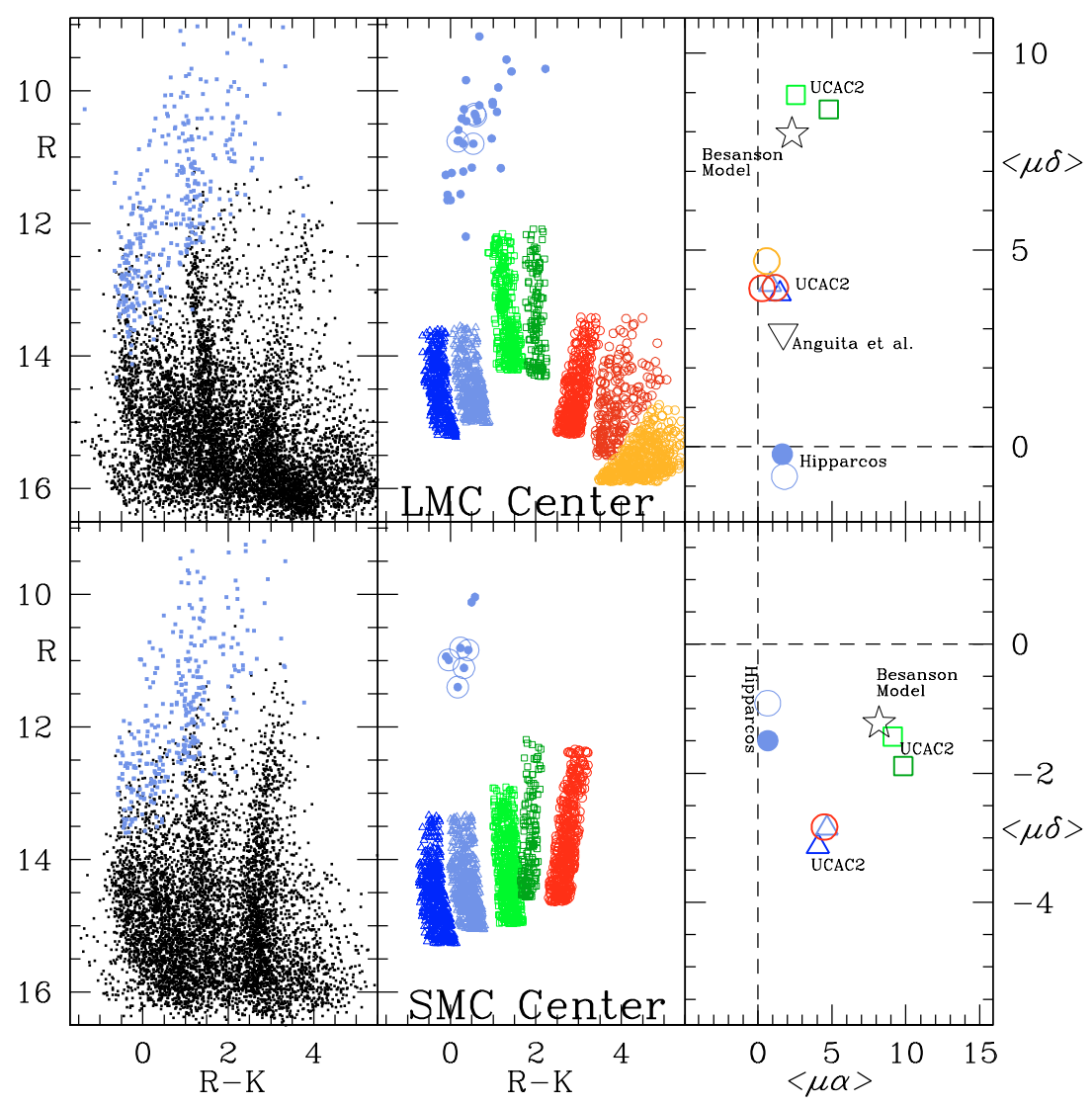

Fig. 1. Left panels display UCAC2/2MASS $R,(R-K)$ color-magnitude diagrams of the LMC and SMC. Dark symbols are used for stars whose pm originates only from the AC2000.2 catalog, while light symbols have a pm coming from a number of catalogs, including Hipparcos. Middle panels highlight the extracted populations: the two vertical sequences around $(R-K) \simeq 1.5$ are Galactic disk stars, the rest are LMC/SMC populations. Dots brighter than $R \sim 12.0$ are Hipparcos samples and tabled in Kroupa \& Bastian (1997). Those plotted as open circles fall within the examined $1^{\circ}$ radius UCAC2 field. Right panels show the mean proper motion of the selected populations in the $\langle\mu \alpha, \mu \delta\rangle$ plane. The different symbols correspond to those in the middle panels. To these we added, an upside down open triangle marking the ALP00 value, while an open starred symbols shows the expected mean pm of Galactic disk stars (as derived from the Besançon simulation).

and $\simeq(4.44,-2.92)$ mas/yr based on $\sim 1200$ stars for the SMC. Lastly, we searched for possible variations of the mean pm across the LMC. However, for all considered fields, the resulting pm in declination was always larger than Hipparcoslike values. In particular, we were able to recover/confirm the ALP00 pm value of their 2 fields centered at $5^{\mathrm{h}} 57^{\mathrm{m}}$ and $5^{\mathrm{h}} 58^{\mathrm{m}}$. Thus, the inconsistency of UCAC2 data is found all over the LMC.

One may be surprised by the high pm values obtained for the Galactic populations. In this regard, we note that the derived pm of Galactic populations is very well reproduced by the Besançon Galactic model simulations. The mean model pm was computed on the synthetic color-magnitude diagrams for the two vertical sequences and is plotted as an open-starred symbol in both the right-most panels. We emphasize that these are model expectations, meant only to test the reliability of UCAC2 values for the disk stars. The agreement between UCAC2 and Besançon however remains quite significant.

\section{The UCAC2-Hipparcos discrepancy}

Unveiling the origin of the UCAC2-Hipparcos discrepancy is quite a difficult task since it may be due to many different sources of systematic errors. In the following we list a number of possible causes.

- Magnitude/color dependencies: the LMC/SMC UCAC2/2MASS selected populations span $\sim 5.0$ mag in $(R-K)$ showing no appreciable dependence on color. Furthermore, dividing the 5 selected populations in magnitude bins did not result in any appreciable variation of the mean pm. Hence, although the presence of residual color/magnitude terms in any pm analysis is almost unavoidable (Platais et al. 2003), these seem not to be significant enough to explain the discrepancy.

- Zone systematics: in Zacharias et al. (2004) pm comparisons in the LMC field were computed for a sample of each of 200 stars. This star by star comparison yielded a difference of $\langle\Delta \mu \alpha, \Delta \mu \delta\rangle=(+0.2,-1.0)$ mas when compared to the average value of van der Marel et al. (2002). This is clearly not enough to explain the $\langle\Delta \mu \delta\rangle \simeq 4.0$ mas Hipparcos-UCAC2 difference. Zacharias et al. also checked for systematic differences in the UCAC2 data as a function of declination zone (their Table 8), again not showing any significant systematic effect. 
- Galactic contamination in the Hipparcos sample: the Hipparcos sample has been selected on the the basis of radial velocity. These are bright, young O/B LMC/SMC members which on Hipparcos color-magnitude diagrams (see also Fig. 1) are clearly limited to the brightest regions $(R \leq 12.00)$, and in a narrow color range $0 \leq(R-K) \leq 1.5$. In this color-magnitude region some contamination by nearby (within $1 \mathrm{kpc}$ ) disk main sequence stars is predicted in the Besançon photometric/kinematic simulation. However the expected radial velocity of these simulated stars is well below $100 \mathrm{~km} \mathrm{~s}^{-1}$ implying that no contaminants should be present in the Hipparcos LMC/SMC sample.

- Hipparcos $O / B$ LMC stars selection effects: the Hipparcos LMC stars $(V \geq 10.5)$ are actually far below the $100 \%$ completeness magnitude limit of Hipparcos $(V \simeq 9)$, and are located in a color-magnitude range where the completeness rapidly falls to zero around $V \simeq 13$. Moreover, these stars are projected on the main body of the LMC and are surrounded by a rich and crowded background that could lead to an incorrect centroiding measurement.

- O/B spectral type dependencies in Hipparcos: a recent detailed analysis by Schroeder et al. (2004) of the Hipparcos parallaxes of Galactic O/B stars pointed out that these are heavily erroneous leading to absolute magnitude estimates up to 5 magnitudes fainter. On the other hand, Pan et al. (2004) identified a duplicity in the star Atlas affecting the centroiding of Hipparcos, and suggested this as the cause of the anomalous (10\% smaller than accepted) Hipparcos distance for the Pleiades. A bias in the parallaxes of bright $\mathrm{O} / \mathrm{B}$ stars in the Pleiades has also been confirmed recently by Soderblom et al. (2004). It is not clear to us how wrong parallaxes can affect proper motion determination, however we note that Hipparcos gives non-negative parallaxes for most of the LMC stars. This is much larger than the $e x$ pected value for the LMC.

In summary, we are unable to find a consistent explanation as to why there exists a discrepancy between the Hipparcos and UCAC2 pm measurements. Any a priori comparison of the two catalogs would certainly favor the UCAC2 as the data source on which to base a pm determination of the LMC. Indeed: $(i)$ the large number statistics involved in the UCAC2 LMC/SMC measurements; (ii) the large time baseline for the pm measurements; (iii) the reliability of UCAC2 in determining the pm for the Sagittarius dwarf; (iv) the excellent consistency of the mean pm of different LMC/SMC populations with different colors and magnitudes; and $(v)$ the good reproduction of the mean $\mathrm{pm}$ of the Galactic populations in the LMC/SMC fields, are all evidence in favor of the UCAC2 measurements with respect to the limited (in magnitude and spectral class) Hipparcos sample.

\section{Discussion: Implications of the UCAC2 proper motion}

Serious problems arise when one replaces the Hipparcos with the UCAC2 value for the LMC proper motion. Indeed, the physical implications of UCAC2/ALP00 pm contrast with our present-day understanding of the Local Group in general, and the Cloud-Galaxy interaction in particular. All methods used to measure the Clouds' transverse velocity $\left(v_{\mathrm{t}}\right)$ argue against the UCAC2 pm values. Applying Eq. (55) of van der Marel et al. (2002) for a Hipparcoslike $\mathrm{pm}$ value, the resulting three-dimensional space velocity for the LMC is $\vec{v}_{\text {LMC }}=(-56,-219,186) \mathrm{km} \mathrm{s}^{-1}$, while the corresponding total, tangential and radial velocities are $(293,281,84) \mathrm{km} \mathrm{s}^{-1}$, in the Galactocentric rest frame. On the other hand, applying the UCAC2 LMC pm gives $\vec{v}_{\text {LMC }}=$ $(-974,-195,-113) \mathrm{km} \mathrm{s}^{-1}$, with the same velocities being $(1001,971,241) \mathrm{km} \mathrm{s}^{-1}$. Similarly, the UCAC2 pm of the SMC would imply $\vec{v}_{\mathrm{SMC}}=(-634,-974,475) \mathrm{km} \mathrm{s}^{-1}$, and $(1256,1251,103) \mathrm{km} \mathrm{s}^{-1}$. These UCAC2-based values are hard to reconcile with our current understanding of the:

- LMC rotation: the UCAC2 implied tangential velocity of the $\mathrm{LMC}, 971 \mathrm{~km} \mathrm{~s}^{-1}$, is so large that, if present, should appear as in a prominent solid-body rotation component as expected from the large projected dimensions in the sky of the LMC. There is no evidence of such a high component (see van der Marel 2002).

- Local Group: in the Galactocentric rest frame, there is no evidence in Local Group or nearby groups of galaxies of such extreme velocities. As recently shown by Karachentsev (2005), the velocity dispersion of dwarf galaxies under the influence of the Milky Way is $\sigma_{v r} \simeq$ $86 \mathrm{~km} \mathrm{~s}^{-1}$. Similar values of the velocity dispersion are found in other nearby groups like Andromeda, CenA, M81/82 etc., indicating that no peculiar motions are present. The LMC radial velocity based on UCAC2 is almost $\simeq 3 \times$ this typical velocity dispersion, i.e. much higher than the escape velocity.

- Magellanic Stream: the UCAC2 LMC transverse velocity puts it at $\sim 90^{\circ}$ from the direction of the Magellanic Stream. Although we still do not know why the stream is starless, it is hard to envisage how this relatively young feature survives at right angles to the motion of the LMC (see Mastropietro et al. 2004, for recent modeling).

- Magellanic Bridge: analysis of the Magellanic Stream and Bridge shows evidence that the Bridge is old (Putman et al. 2003). This strengthens the idea that the LMC and SMC have jointly orbitted around the Galaxy at least for the past Gyr or so. However, this observational evidence is hard to reconcile with the hypothetical UCAC2 scenario where there exists a relative velocity between the LMC and SMC of the order of $1033 \mathrm{~km} \mathrm{~s}^{-1}$.

A scenario with the LMC traveling at a high velocity (first passage) in the vicinity of the Milky Way seems also to contradict recent observational (Cole et al. 2005) and theoretical (Bekki \& Chiba 2005) studies that successfully reproduce the observed morphology, dynamics and formation histories of the LMC assuming it has been in tidal interaction with the SMC and the Milky Way. The only viable scenario that allows for the UCAC2/ALP00 LMC pm is one in which the Milky Way is more massive (within $50 \mathrm{kpc}$ ) than $3 \div 4 \times 10^{12} M_{\odot}$ as suggested by Méndez et al. (1999). Interestingly, the Méndez et al. modeling is based on a proper motion analysis of 30000 stars from 
the Southern Proper-Motion (SPM) survey, and is consistent with the Anguita et al. pm value of the LMC. Nevertheless, the Méndez et al. Milky Way total mass estimate remains much larger than those reported in recent studies, e.g. Sakamoto, Chiba \& Beers (2003) who estimate the mass of the Galaxy (within $50 \mathrm{kpc}$ ) to be $5.5 \times 10^{11} M_{\odot}$.

In conclusion, the UCAC2 data may have been valuable in determining the pm of the Sagittarius dwarf at $25 \mathrm{kpc}$ and in the direction of the Canis Major over-density at $\simeq 8 \mathrm{kpc}$, yet the UCAC2 $\mathrm{pm}$ data are hard to reconcile with our present day understanding of the Cloud-Galaxy interaction. Given that our study has failed to identify sources of systematic errors, the UCAC2/Hipparcos inconsistency remains unsolved and awaits high-accuracy HST, GAIA and SIM proper motion measurements.

Acknowledgements. We are indebted to the anonymous referee whose comments have improved this paper. We thank Giampaolo Piotto and Luigi Bedin for helpful comments on the manuscript. Y.M. acknowledges financial support by MIUR under the program PRIN2003. Z.S. acknowledges financial support by MIUR PRIN2002: "Stellar Populations in the Local Group as a Tool to Understand Galaxy Formation and Evolution".

\section{References}

Alcock, C., Allsman, R. A., Alves, D. R., et al. 2000, AJ, 119, 2194 Anguita, C., Loyola, P., \& Pedreros, M. H. 2000, AJ, 120, 845

Bekki, K., \& Chiba, M. 2005, MNRAS, 356, 680

Cole, A., Tolstoy, E., Gallagher, J. S., \& Smecker-Hane, T. A. 2005 , AJ, 129, 1465

Cutri, R. M., Skrutskie, M. F., van Dyk, S., et al. 2003, VizieR Online Data Catalog, 2246, 0
Dinescu, D. I., Girard, T. M., van Altena, W. F., \& Lopez, C. E. 2004, abstract of congress, Astrometry in the Age of the Next Generation of Large Telescopes, held in Flagstaff, October 2004

Drake, A., et al. 2002, 199th Meeting of the American Astronomical Society, 52.05

Girard, T. M., Dinescu, D., van Altena, W., et al. 2004, AJ, 127, 3060

Irwin, M., Ibata, Gilmore, G., Wyse, \& Suntzeff, N. 1996, Formation of the Galactic Halo. Inside and Out, ASP Conf. Ser., 92, 84

Karachentsev, I. D. 2005, AJ, 129, 178

Kroupa, P., Röser, S., \& Bastian, U. 1994, MNRAS, 266, 412

Kroupa, P., \& Bastian, U. 1997, New Astron., 2, 77

Mastropietro, C., Moore, B., Mayer, L., Wadsley, J., \& Stadel, J., MNRAS [arXiv: astro-ph/0412312]

Méndez, R. A., Platais, I., Girard, T. M., Kozhurina-Platais, V., \& van Altena, W. F. 1999, ApJ, 524, L39

Momany, Y., Zaggia, S., Bonifacio, Piotto, G., De Angeli, Bedin, \& Carraro, G. 2004, A\&A, 421, L29

Nikolaev, S., \& Weinberg, M. D. 2000, ApJ, 542, 804

Pan, X., Shao, M., \& Kilkarni, S. R. 2004, Nature, 427, 326

Pedreros, M. H., Anguita, C., \& Maza, J. 2002, AJ, 123, 1971

Platais, I., Wyse, R., Hebb, Lee, \& Rey, S. 2003, ApJ, 591, L127

Sakamoto, T., Chiba, M., \& Beers, T. C. 2003, A\&A, 397, 899

Schroeder, S., Kaper, Lamers, H., Brown, A. 2004, A\&A, 428, 149

Soderblom, D., et al. 2004, American Astronomical Society Meeting, 204

Urban, S., Corbin, T., Wycoff, G., Hoeg, \& Makarov, V. 2001, VizieR Online Data Catalog, 1275, 0

Urban, S., Corbin, T., Wycoff, G., et al. 1998, AJ, 115, 1212

van der Marel, R. P., Alves, D. R., Hardy, E., \& Suntzeff, N. B. 2002, AJ, 124, 2639

van der Marel, R. P. 2004, in The Local Group as an Astrophysical Laboratory, Proc. May 2003 STScI Symp., ed. M. Livio (Cambridge, UK: Cambridge University Press)

Putman, M. E., Staveley-Smith, L., Freeman, K. C., Gibson, B. K., \& Barnes, D. G. 2003, ApJ, 586, 170

Zacharias, N., Urban, S., Zacharias, M., et al. 2004, AJ, 127, 3043 